\title{
Kidnapped Laser-Scanner for Evaluation of RFEC Tool
}

\author{
Raphael Falque, Teresa Vidal-Calleja, and Jaime Valls Miro \\ University of Technology Sydney, Australia \\ Raphael.H.Guenot-Falque@student.uts.edu.au,Teresa.VidalCalleja@uts.edu.au,Jaime.VallsMiro@uts.edu.au
}

\begin{abstract}
An algorithm is proposed for matching data from different sensing modalities. The problem is formalised as a kidnapped robot problem, where Bayesian fusion is used to find the most likely location where both modalities agree. The key idea of our algorithm is to model the correlation between the two modalities as a likelihood used to update a location prior. Data, in this case, is represented as 2.5D thickness maps from a laser scanner and a Remote Field Eddy Current (RFEC) tool, used in non-destructive testing to assess the condition of infrastructures. The laser data is limited, while RFEC data is continuous. Given some prior in location, the aim is to find the 2.5D thickness map from the laser that corresponds to the RFEC data, which should be noted is highly noisy. Real data from CCTV inspections of water pipes are used to validate the proposed approach.
\end{abstract}

Keywords : Remote Field Eddy Current, Kidnapped robot problem, map-matching, location prior

\section{INTRODUCTION}

When a robot loses the track of its position on a given map is commonly referred as the "kidnapped robot problem" [4]. Once the robot is lost, it has to explore the environment by comparing the processed sensor measurements to the known information to re-localise itself in the map. In this paper, we consider one step of this re-localisation problem, where a noisy global map is given together with local robot information and a prior on the location. We propose a methodology to locate "the robot" (in our case a pipe segment) in the global map (built using data from a sensing modality), given a locally referred measurements (obtain with a different sensing modality) and a rough estimate on where to search for possible matches.

More practically, the global map is built with an inline RFEC inspection tool, shown in Fig. 1(a). The tool travels inside an underground pipeline allowing to assess its condition. This type of inspection tools collects data that directly relates to the thickness of the pipeline. All data are associated with a location using wheel odometers embedded on the tool. This allows creating a $2.5 \mathrm{D}$ map, similar to an elevation map, where the two dimensions represent the axial and circumferential locations and the half dimension represents the thickness of the pipeline. In our experiment, the global map consists of $1 \mathrm{~km}$ of pipeline dedicated for research purposes.

The locally referred measurements have been obtained by exhuming small $(\sim 1 \mathrm{~m})$ pipe sections as shown in Fig. 1(b). These sections are cleaned and analysed with a three dimensional (3D) laser scanner as shown in Fig. 1(c).
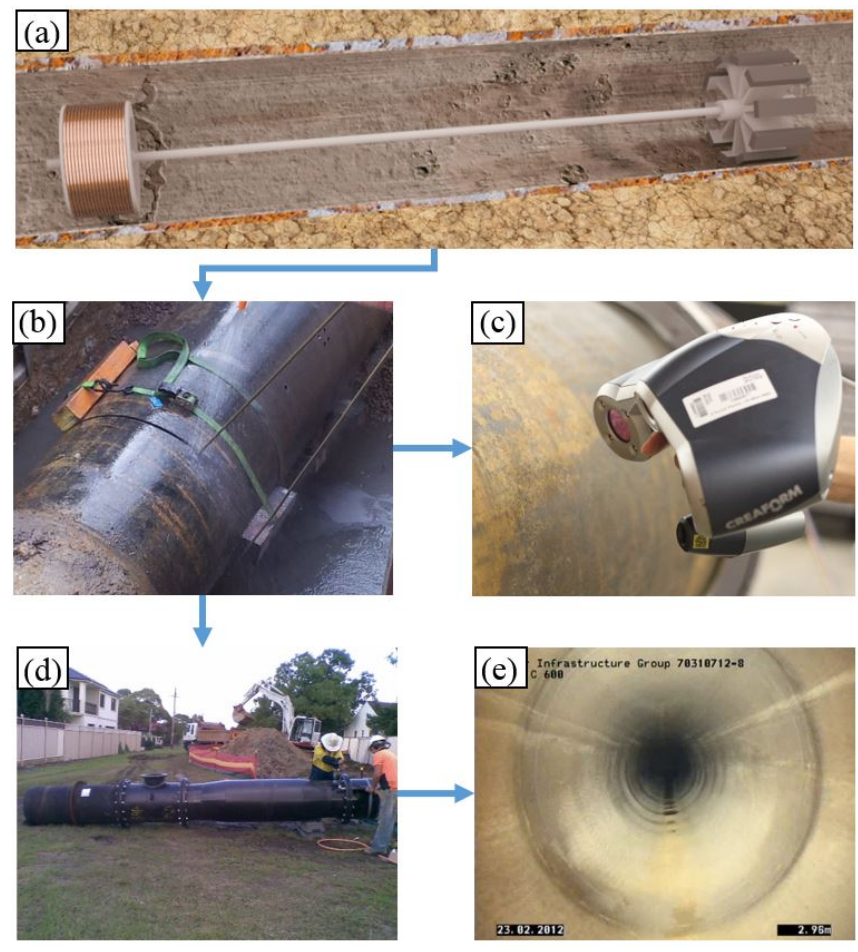

Fig. 1: Overview of the data acquisition. (a) The pipeline is inspected using Remote Field Eddy Current (RFEC) tool, (b) some pipes section are extracted (c) to be assessed using laser scanner. (d) The extracted pipes are replaced by new pipe sections and (e) a CCTV inspection is run through the pipe at the end of the process.

After transforming the geometry captured by the laser scanner into thickness [10], we obtain a local, high resolution, and accurate $2.5 \mathrm{D}$ map. Note that exhuming a part of the pipeline, instead of introducing the robot into the pipeline, results in the same theoretical formulation of the kidnapped robot problem since we consider only one iteration for relocalisation. Since the robot measurements are transformed into a $2.5 \mathrm{D}$ thickness map of the extracted pipe section, they are referred as local maps for the rest of the paper. In our current research, these local maps are used as ground truth for evaluation of the RFEC technology.

Both, the data from the RFEC tool and laser scanner, have to be aligned to be compared to each other. It is possible to do a rough alignment using the odometer information while travelling inside the pipe and the distance measured 
above the ground when excavating the pipe sample. However, due to the inherent drift in the odometry, the lack of loop closures, and the inaccuracy of the manual procedure to measure distances above the ground, alignment of only $1 \mathrm{~m}$ of data results quite challenging. Furthermore, as the pipeline are buried deep underground and the terrain changes, the distances measured inside the pipe and the ones measured above the ground are different. A common advice from technology providers of REFC tools is to dig within a window of $20 \mathrm{~m}$ to get a specific $3 \mathrm{~m}$ long pipe section.

Alignment of $2.5 \mathrm{D}$ maps is commonly referred in the literature as template matching, map matching or registration depending on the research field and application. The problem is generally formulated as the minimisation of a distance between the two maps/images. In the field of medical imaging, authors often deal with different modalities such as MR and ultrasound, and use metrics such as Mutual Information (MI) for measuring the dissimilarity between the different data for 2D [14] and 3D images [8]. This problem is also present for localisation in the robotic field, where multi-modal map matching techniques are commonly used for localisation in robotics [15], [7]. In the problem tackled in this paper, we also deal with different sensing modalities.

The MI metric, however, sometimes shows unexpected results, producing better scores for wrongly registered maps than for properly registered ones [11]. This problem can be solved by using Normalised Mutual Information (NMI), or using problem-designed metrics. For example, in the case of a known linear relationship between the sensor modalities, Pearson correlation [9] is simple and considerably reduces the computation cost of the algorithm.

In our case, due to the noisy nature of the global map, these methods shows poor results. Luckily, the pipeline contains structural elements such as joints. They are both visible within the RFEC data and in the exposed pipe section before exhumation. In our experimentation, distance measurements were taken between the exhumed pipe section and these structural elements allowing us to have a strong location prior when aligning the data. Similarly to [1], the prior is incorporated in the registration approach using a Bayesian approach.

The performance of the proposed method is evaluated using the location obtained by visually inspecting the pipeline after exhumation of all pipe sections. The pipe sections are replaced by new pipes (shown in Fig. 1(d)) made from different material, making possible to find them with a CCTV inspection (shown in Fig. 1(d)). Finally, the structural elements are used to align the video with the data captured by the RFEC tool. This provided us with the exact locations of the exhumed pipe section with respect to the global map given by the RFEC tool. We present results here that show the validity of the approach.

\section{MAPPING OF THE PIPELINE}

The global map $m$ is built by scanning the entire pipeline using an in-line RFEC tool. The tool generates a magnetic field while travelling through the one-kilometre pipeline. The ferromagnetic medium of the cast-iron pipe interacts with the magnetic field and the response of the excitation is recorded by the tool.

The magnetic properties recorded are the log-amplitude and the phase shift of the magnetic field, which have been proved to have a linear relationship with the pipe thickness [2]. Simultaneously to the acquisition of the magnetic field properties, the displacement of the tool is recorded by three embedded wheel encoders. The information obtained from the wheel encoders are merged to get the odometry information.

The data collected is highly noisy and requires a thorough filtering process to obtain the correlation with the thickness of the pipe. Faulty and miss-calibrated sensors are present in the data.

The missing sensors are interpolated using the neighbouring sensors and the miss-calibration is corrected using a Butterworth filter [3] in the Fourier space. An example of this filtering is displayed in Fig. 2.

Furthermore to the simple filtering, a background removal technique, shown in Fig. 3, is applied to the RFEC signal to counteract the effect of the magnetic field passing twice through the pipe's wall. This approach is explained in [5].

The inspection performed with the RFEC tool allows us to produce a global map with a grid size of $2 \times 37 \mathrm{~mm}$. This resolution is limited by the design of the tool itself and the sampling rate of the signal.

\section{LOCAL MAP}

The local map $m_{\text {local }}$ is defined by the thickness map of a pipe section, which is usually a one-meter long cylinder. To create this thickness map, we use a commercial highresolution 3D laser scanner shown in Fig. 4.

After scanning, the raw 3D model acquired by the laser scanner contains outliers and needs to be processed to be transformed into the same state space as the map $m$, which is a $2.5 \mathrm{D}$ thickness map. The model is processed with a stateof-the-art 3D point cloud algorithms detailed in [10]. After removing the outliers, the $3 \mathrm{D}$ point cloud is then ray-casted to obtain the thickness map of a $1.5 \mathrm{~mm}$ uniform sampling.

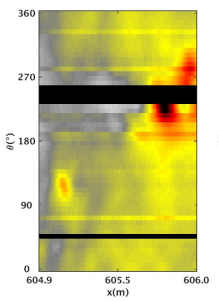

(a)

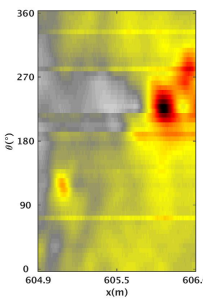

(b)

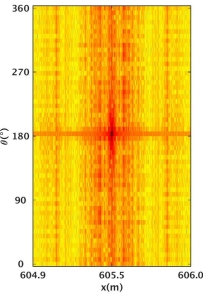

(c)

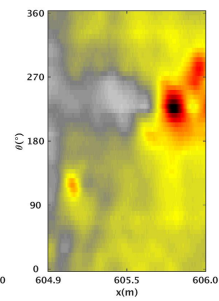

(d)
Fig. 2: (a) Raw data have missing and miscalibrated sensors. (b) The missing sensors are interpolated using the surrounding sensors. (c) The data are then transformed into the Fourier space. (d) The lack of calibration is solved by applying a Butterworth filter in the Fourier space. 

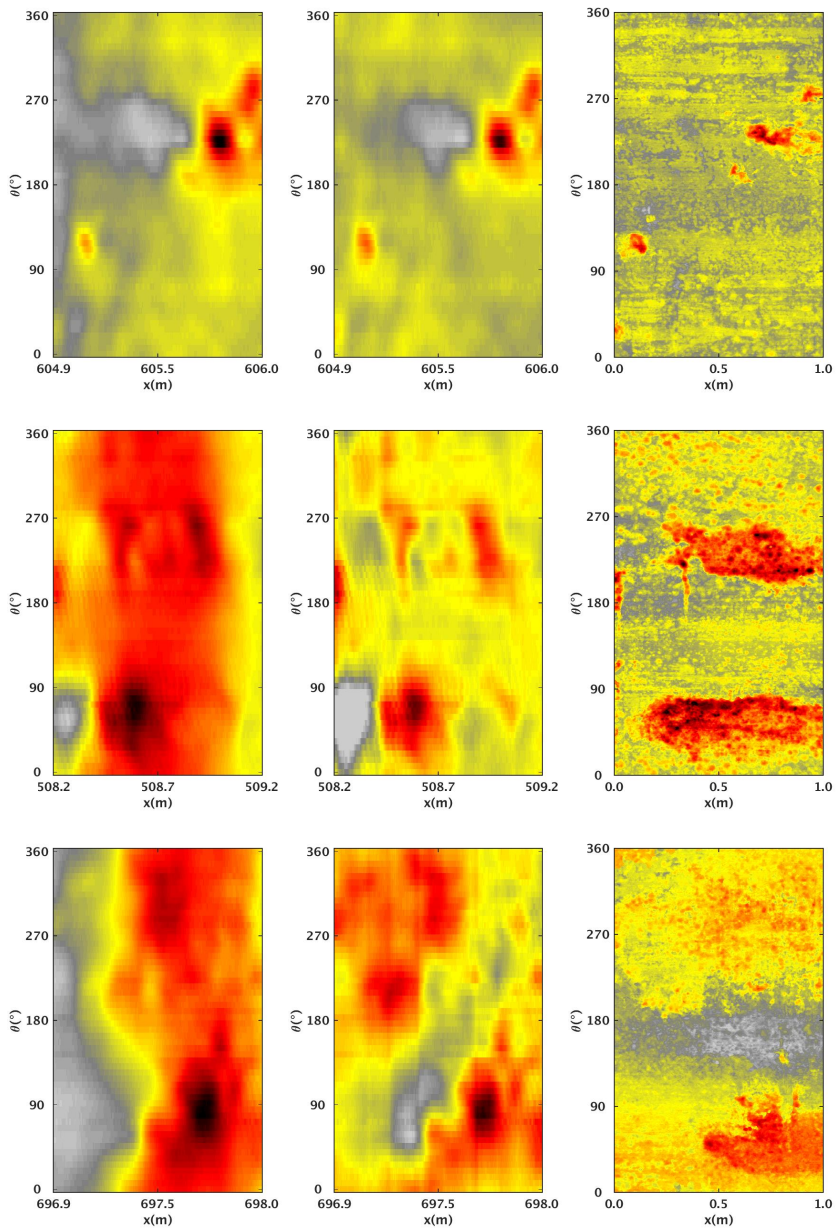

Fig. 3: The calibrated RFEC signal, shown in the first row, is processed with a background removal algorithm to obtain the data shown in the middle row. In case of high source of noise such as the two last lines, the data still presents poor correlation with the laser scans, shown in the last row.

\section{MAP-MATCHING}

Let the state $\mathbf{x}$ be the location of the laser scanner within the global map

$$
\mathbf{x}=\left[\begin{array}{l}
x \\
\theta
\end{array}\right],
$$

where $x$ is the axial position of the laser scan and $\theta$ is the circumferential shift. The aim is to transform both maps $m$ and $m_{\text {local }}$ into a likelihood space using a correlation-based measurement model [12].

\section{A. Matching the grid space}

In order to compute the correlation function, the maps need to be defined at the same resolution. The local map $m_{\text {local }}$, which has a much larger resolution, is down-sampled to fit the grid size of the map $m$.

These maps are defined in cylindrical coordinates, but they are manipulated as matrices that do not account for the cylindrical wrapping of the data. It is however interesting to consider the cylindrical properties of the maps while matching the grid-size of the data. We create an overlap of the data in the $2 \mathrm{D}$ matrix which represent the map $m_{\text {local }}$. More formally, from the original $m_{\text {local }}$ of size $X \times \Theta$, we create a temporary matrix $m_{\text {temp }}$ of size $X \times \Theta+2$ defined as follows,

$$
m_{\text {temp }}=\left[\begin{array}{c:ccc:c}
m_{1, \Theta} & m_{1,1} & \cdots & m_{1, \Theta} & m_{1,1} \\
\vdots & \vdots & \ddots & \vdots & \vdots \\
m_{X, \Theta} & m_{X, 1} & \cdots & m_{X, \Theta} & m_{X, 1}
\end{array}\right] .
$$

To resize the image $m_{X 1, \Theta 1}$ to the size $X 2 \times \Theta 2$ we use a proportion factor $\alpha_{x}$ on the radial axis, define by

$$
\alpha_{x}=\operatorname{round}\left(\Theta 2 \frac{\Theta 1+2}{\Theta 1}\right) .
$$

The additional columns created are then deleted to match the size $X 2 \times \Theta 2$. This method gives a better approximation while interpolating the values located near the edges of the pipe (which correspond to the section on the radial axis).

\section{B. Map correlation function}

Following the idea of the correlation-based model described in [12] (Chapter 6), let the map correlation function that compares the global and local maps be

$$
\rho_{m, m_{\text {local }}, \mathbf{x}}=\frac{\sum_{x, \theta}\left(m_{x, \theta}-\bar{m}\right)\left(m_{x, \theta, \text { local }}-\bar{m}\right)}{\sqrt{\sum_{x, \theta}\left(m_{x, \theta}(\mathbf{x})-\bar{m}\right)^{2} \cdot \sum_{x, \theta}\left(m_{x, \theta, \text { local }}(\mathbf{x})-\bar{m}\right)^{2}}},
$$

were $\bar{m}$ is the average map value expressed as

$$
\bar{m}=\frac{1}{N} \sum_{x, \theta}\left(m_{x, \theta}+m_{x, \theta, \text { local }}\right),
$$

and $N$ is the number of elements present in both $m$ and $m_{\text {local }}$.

Also, let the map correlation function be expressed as a probability

$$
p\left(m_{\text {local }} \mid \mathbf{x}, m\right)=\max \left\{\rho_{m, m_{\text {local }}, \mathbf{x}}, 0\right\} .
$$

Then as local map is generated from a single range of measurement $z$, we can consider $p\left(m_{\text {local }} \mid \mathbf{x}, m\right)$ as a measurement probability $p(z \mid \mathbf{x}, m)$.

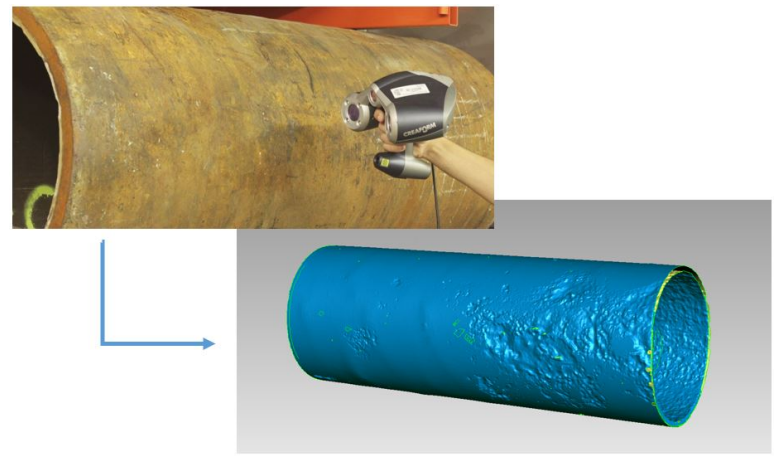

Fig. 4: Generation of a 3D model of the pipe using a laser scanner. The $3 \mathrm{D}$ model is then transformed into a $2.5 \mathrm{D}$ thickness map. 

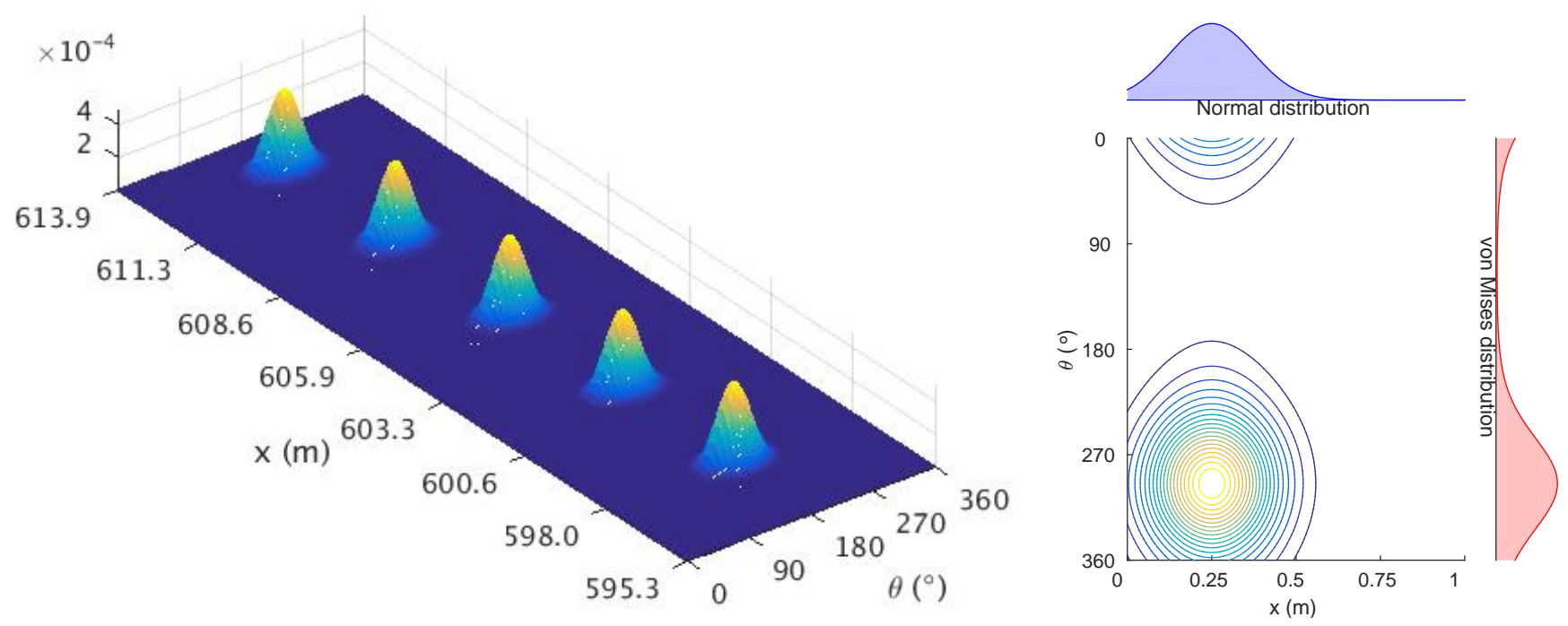

Fig. 5: The prior map, on the left, is defined as a mixture of Gauss von Mises (GVM) distributions along the pipe. On the right figure, the parameters $\Theta(\mathrm{x})$ and $\kappa$ have been defined to emphasised the wrapping property of the GVM distribution.

Current practice advices to search at least two pipe sections upstream and downstream from the exhumed pipe section to overcome odometry errors. We perform an exhaustive search of the map-matching on the domains $\operatorname{dom}(x) \times \operatorname{dom}(\theta)$ for all these pipe sections. The search is operated off-line, thus the computation time is not an issue.

\section{LOCATION PRIOR}

The one-kilometre long pipeline is constituted of short pipe sections, which are attached together. These pipe sections are usually $3.6 \mathrm{~m}$ long and the attachments between the pipes are done using Bell and Spigot $(B \& S)$ joints. The $\mathrm{B} \& \mathrm{~S}$ joints are visible in the data acquired by the RFEC tool. Moreover, these joints can be also located visually when the pipes are extracted from the ground, therefore they are a good reference for matching the different data.

In order to get the data aligned, the distance between these $B \& S$ joints and the section extracted have been manually measured. From the RFEC data, an automatic algorithm has been developed to locate the B\&S joints [13]. This algorithm uses a machine learning approach using Support Vector Machine (SVM) to classify the Bell \& Spigot joints, and other construction features.

We use this information to build a representation of the location prior. The prior knowledge is defined as a featurebased map that is a Gauss von Mises (GVM) distribution [6], more formally we define it as a Probability Density Function (PDF),

$$
\mathrm{p}_{\text {local }}(x, \theta)=N(x ; \mu, \sigma) V M(\theta ; \Theta(x), \kappa),
$$

with $N(x ; \mu, \sigma)$ the normal distribution and $V M(\theta ; \Theta(x), \kappa)$ the von Mises distribution, which is an approximation of the wrapped normal distribution,

$$
\begin{array}{r}
N(x ; \mu, \sigma)=\frac{\mathrm{e}^{-\frac{1}{2} \frac{(x-\mu)^{2}}{\sigma^{2}}}}{\sqrt{2 \pi \sigma^{2}}}, \\
V M(\theta ; \Theta(x), \kappa)=\frac{\mathrm{e}^{\kappa \cos (\theta-\Theta(x))}}{2 \pi \mathrm{I}_{0}(\kappa)},
\end{array}
$$

where $\mathrm{I}_{0}(\kappa)$ is a modified Bessel function of the first order, $\sigma$ and $\kappa$ relate to the variance of each distribution, and $\mu$ and $\Theta(x)$ are the center of each distribution $(\Theta(x)$ is a function of $\mathrm{x}$ to account for the cross correlation between $\mathrm{x}$ and $\theta$ ).

On the axial direction, $\sigma$ is defined with an uncertainty related to the size of a B\&S joint. On the circumferential direction, the rolling of the tool within the pipe is supposed to be corrected, however, a small drift will lead to a significant change after a long distance. Therefore, we define $\kappa$ as an equivalent to a $\sigma$ of $30^{\circ}$ on the circumferential direction.

A similar PDF is defined for each section of the pipe. The set of PDF is then combined as a Gaussian mixture,

$$
\mathrm{p}(\mathbf{x})=\frac{1}{\sum_{l} \psi_{l}} \sum_{l} \psi_{l} \mathrm{p}_{\text {local }}(\mathrm{x}, \theta),
$$

where $\psi_{l}$ is the weight of each $\mathrm{p}_{\text {local }}(\mathrm{x}, \theta)$.

\section{BAYESIAN FUSION OF THE LOCATION PRIOR AND SENSOR MEASUREMENT}

The location prior $p(\mathbf{x})$ is fused to the measurement likelihood $p(z \mid \mathbf{x}, m)$ using the Bayes rule

$$
\mathrm{p}(\mathbf{x} \mid z)=\frac{p(z \mid \mathbf{x}, m) \times p(\mathbf{x})}{p(z)} .
$$

The location of the best match is defined by $\operatorname{argmax}\{\mathrm{p}(\mathbf{x} \mid z)\}$, known as the maximum a posteriori (MAP) estimator. Since $\mathrm{p}(z)$ is a normalising constant, which does not change the result of the argmax function, we do not consider it. 


\section{RESULTS AND DISCUSSION}

The exhumation and analysis of the pipe sections is a costly and laborious exercise, which requires actual construction work. Therefore, the amount of data available (for local maps) is limited to a few pipe sections. For this analysis, we consider nine pipe segments of $1 \mathrm{~m}$ to be compared with five $3.6 \mathrm{~m}$ sections each.

\section{A. Validation of the methodology}

The validation of the methodology is performed by analysing a CCTV inspection of the pipeline. The exhumed pipe sections have been replaced by new sections made out of different material, which has different coloration. Therefore, locating these pipe sections within the CCTV data results in a trivial task.

The data from the CCTV inspection and the global map are aligned using the visible $B \& S$ joints. The drift from the RFEC tool odometry can be corrected iteratively after each joint. After alignment of the data, we obtain an accurate localisation of the exhumed pipes.

Fig. 6, 7, and 8 show the performance of the proposed algorithm in different scenarios. Figs. 6 and 7 show successful matching using the MAP estimator on the posterior. The proposed methodology shows robust performance in a noisy environment (Fig. 7). When comparing the RFEC signal and the laser scans at the correct location (second line in Fig. 3), the important source of noise results in poor correlation between both data.

Fig. 8 illustrates the situation of high noise in the global map. The posterior still show a bimodal behaviour which include the correct match and provide better information than without using location prior.

The performance of the methodology used on the full dataset is presented in confusion matrix of Table I. As the table shows, the algorithm has $80 \%$ accuracy. The high number of false positives is due to the lack of features on some of the pipes samples. The lack of features produces the bimodal behaviour as explain above. The algorithm, however, produced a small number of false negatives that in practical terms is more important than the false positives. Note that false negatives represent the times the algorithm matched the map with the incorrect location, while the false positives, in this case, represent the ambiguity produced by the algorithm.

TABLE I: Confusion matrix summarising the results of the map matching.

Predicted as

at the location out the location

\begin{tabular}{ccc}
\hline At the location & 8 & 1 \\
\hline Outside the location & 8 & 28 \\
\hline
\end{tabular}

\section{B. Discussion}

Due to the nature of the RFEC technology, the global map $m$ is very noisy. In the presence of features (defects in the pipe), the algorithm performs well as the global map is not too noisy. Failures such as the one in Fig. 8 happens when the ratio of signal to noise is too low. Even in this noisy condition, the posterior shows a bimodal behavior which proves a clear improvement compared to the correlation map only. The failure in the matching of the algorithm happens when the ratio signal to noise is too low and there are no features (defects) on the map.

Using the correlation map function offers the advantage to perform the search on $\theta$, which would not have been possible with either MI or NMI since these distances are based on the entropy of an image and do not account for pixels location. Furthermore, the correlation map function is much faster to compute than MI and NMI, which allows us to perform an exhaustive search easily.

The computation efficiency was not important in our experiment since the algorithm was run off-line, however, a threshold on the location prior could be used to reduce the search space.

The algorithm can be generalised to some other scenarios of global maps and local measurements defined in a cylindrical space. This methodology has been applied for aligning scans produced by an Magnetic Flux Leakage (MFL) tool with ground truth, showing promising results.

\section{CONCLUSION}

The use of a strong location prior allows to constrain a standard map matching approach. The proposed method accounts for the cylindrical geometry of the data and allows finding the good location of the scanned pipe for both axial and circumferential direction, which is not possible with an MI-based map matching.

Up to a certain level of noise, we can recover the proper location of the extracted pipe and for a high level of noise, the location prior still improve the results.

The use of a correlation map function reduces the computation time and allows a multi-modal comparison in case of a linear relationship. In future work, we will look into the use of a gradient-based optimisation to overcome the need of exhaustive search for a more computationally efficient methodology.

\section{ACKNOWLEDGMENT}

This publication is an outcome from the Critical Pipes Project funded by Sydney Water Corporation, Water Research Foundation of the USA, Melbourne Water, Water Corporation (WA), UK Water Industry Research Ltd, South Australia Water Corporation, South East Water, Hunter Water Corporation, City West Water, Monash University, University of Technology Sydney and University of Newcastle. The research partners are Monash University (lead), University of Technology Sydney and University of Newcastle. 

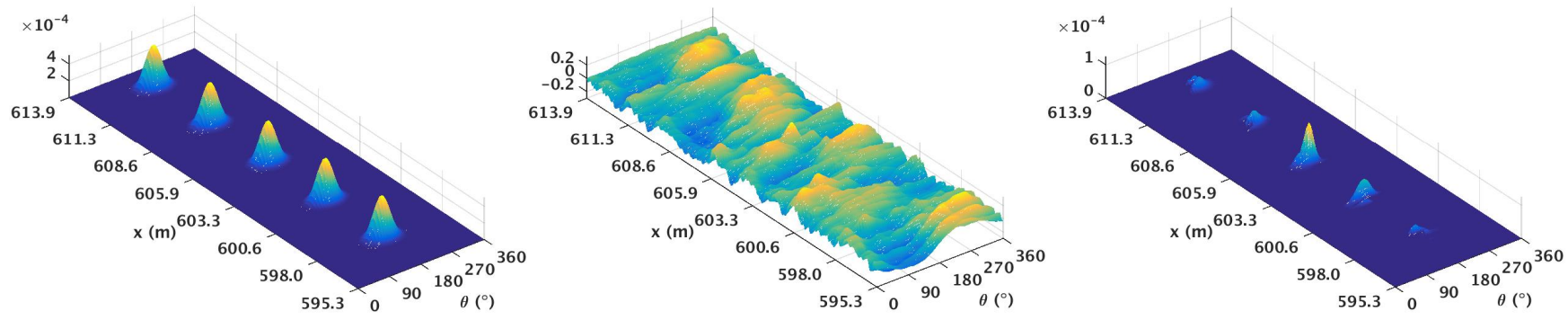

Fig. 6: Example of successful matching with low noise data displayed in the first line of Fig. 3. The left figure shows the location prior. The map correlation function, in the middle, shows a local maximum in the location of the correct match. The Bayesian fusion with the location prior is shown on the right.
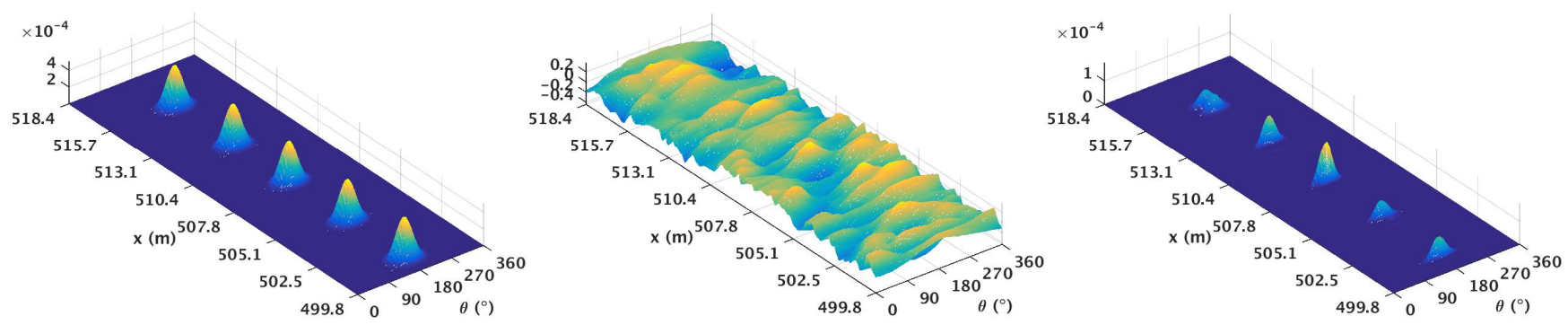

Fig. 7: Example of a successful matching from a noisy data, the RFEC and laser scan data are displayed in the second line of Fig. 3. The uses of the location prior allows to distinguish the local maxima in the correlation map.
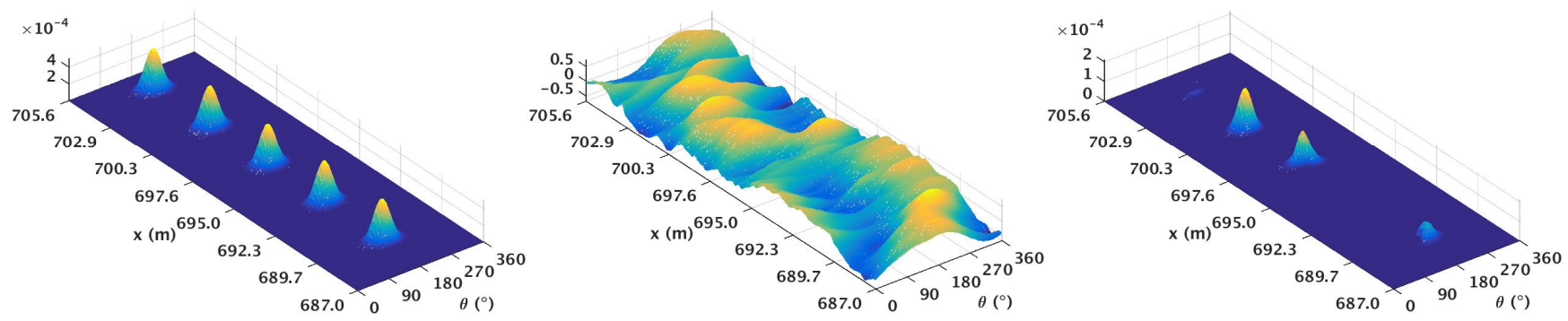

Fig. 8: Example of multi-modal posterior from noisy data, the RFEC and laser scan data are displayed in the last line of Fig. 3. The measurement of the global map contains too much noise to have the successful match using the MAP estimator, however the use of the location prior allow to have much better certainty than using the single map correlation function.

\section{REFERENCES}

[1] J Ashburner, P Neelin, D L Collins, a Evans, and K Friston. Incorporating prior knowledge into image registration. NeuroImage, 6(4):344352, 1997.

[2] David L Atherton. Remote field eddy current inspection. IEEE Transactions on Magnetics, 31(6):4142-4147, 1995.

[3] Stephen Butterworth. On the Theory of filter Amplifiers. Wireless Engineer, 7(6):536-541, 1930.

[4] Sean P Engelson and Drew V Mcdermott. Error Correction in Mobile Robot Map Learning. International Conference on Robotics and Automation, pages 2555-2560, 1992.

[5] Raphael Falque, Teresa Vidal-calleja, Jaime Valls Miro, Daniel C Lingnau, and David E Russell. Background Segmentation to Enhance Remote Field Eddy Current Signals. In Australasian Conference on Robotics and Automation (ACRA), pages 1-9, 2014.

[6] Joshua T Horwood and Aubrey B Poore. Gauss von Mises Distribution for Improved Uncertainty Realism in Space Situational Awareness . SIAM/ASA J. Uncertainty Quantification, 2:276-304, 2014.

[7] Jesse Levinson and Sebastian Thrun. Robust vehicle localization in urban environments using probabilistic maps. 2010 IEEE International Conference on Robotics and Automation, pages 4372-4378, 2010.

[8] F Maes, a Collignon, D Vandermeulen, G Marchal, and P Suetens. Multimodality image registration by maximization of mutual information. IEEE transactions on medical imaging, 16(2):187-198, 1997.
[9] Ziad S. Saad, Daniel R. Glen, Gang Chen, Michael S. Beauchamp, Rutvik Desai, and Robert W. Cox. A new method for improving functional-to-structural MRI alignment using local Pearson correlation. NeuroImage, 44(3):839-848, 2009.

[10] Bradley Skinner, Teresa Vidal-calleja, Jaime Valls Miro, Freek De Bruijn, and Raphael Falque. 3D Point Cloud Upsampling for Accurate Reconstruction of Dense 2.5D Thickness Maps 3D Point Cloud Acquisition. Australian Robotics \& Automation Association, 2014.

[11] C. Studholme, D.L.G. Hill, and D.J. Hawkes. An overlap invariant entropy measure of 3D medical image alignment. Pattern Recognition, 32(1):71-86, 1999.

[12] Sebastian Thrun, Wolfram Burgard, and Dieter Fox. Probabilistic Robotics (Intelligent Robotics and Autonomous Agents series). Intelligent robotics and autonomous agents. The MIT Press, August 2005.

[13] Teresa Vidal-calleja, Jaime Valls Miro, and Fernando Mart. Automatic Detection and Verification of Pipeline Construction Elements with Multi-modal data. In IROS, 2014.

[14] W M Wells, P Viola, H Atsumi, S Nakajima, and R Kikinis. Multimodal volume registration by maximization of mutual information. Medical image analysis, 1(1):35-51, 1996.

[15] Ryan W. Wolcott and Ryan M. Eustice. Visual localization within LIDAR maps for automated urban driving. 2014 IEEE/RSJ International Conference on Intelligent Robots and Systems, (Iros):176-183, September 2014. 